\title{
Detecção de Falhas em um Sistema Rotativo utilizando Sistemas Imunológicos Artificiais
}

\author{
Rodrigo F. B. Lourenço ${ }^{1}$ \\ PPGEM / UNESP, Ilha Solteira, SP; UNIRV, Rio Verde, GO \\ Fabio R. Chavarette ${ }^{2}$ \\ PPGEM / UNESP, Ilha Solteira, SP \\ Roberto Outa ${ }^{3}$ \\ FATEC, Araçatuba, SP \\ Michael D. G. Silva ${ }^{4}$ \\ PPGEM / UNESP, Ilha Solteira, SP \\ Darlan M. da Silva 5 \\ UNIRV, Rio Verde, GO
}

\begin{abstract}
Resumo. É ascendente a pesquisa e desenvolvimento de ferramentas computacionais, baseadas em técnicas de computação inteligente, afim de detectar falhas estruturais em máquinas e equipamentos no mundo inteiro. Essa pesquisa propõe o desenvolvimento de um Sistema de Monitoramento da Integridade Estrutural (do inglês, Structural Health Monitoring-SHM), com base na teoria de Sistemas Imunológicos Artificiais, utilizando-se o Algoritmo de Seleção Negativa (ASN), que determina a identificação e quantificação de falhas de um sistema dinâmico rotativo. O sistema de aquisição de dados utilizado, foi proposto na visão da simplicidade e baixo custo, composto por um microcontrolador Arduíno e um acelerômetro MPU6050. Para aquisição do banco de dados com sinais livres de falhas (sinais próprios), utilizou-se um intervalo de frequência que variou de $2 \mathrm{~Hz} \mathrm{a} 60 \mathrm{~Hz}$, em passos de $2 \mathrm{~Hz}$, contendo 10 sinais de tamanho $(1 \times 4000)$ para cada frequência. O banco de dados detectores (não próprios), foi constituído após a retirada de dois parafusos (um oposto ao outro) da junta flexível, o que gerou o desbalanceamento do sistema. Com base nos critérios de funcionalidade proposto, perante a avaliação da robustez, precisão e eficiência no processo de diagnóstico de falhas no rotor, o algoritmo imunológico artificial (ASN), apresentou excelente desempenho, quando aplicado para seleção e grupamento de amostras (sinais). O algoritmo foi capaz de identificar as falhas do desbalanceamento do sistema, apresentando acertos com $100 \%$ de precisão.
\end{abstract}

Palavras-chave. SHM, AIS, Análise de Vibrações, Bioengenharia, Sistemas Dinâmicos

\section{Introdução}

Apesar das inúmeras vantagens de aplicação dos motores de indução, falhas elétricas e mecânicas representam um desafio particular para a indústria e para os usuários finais desses equipamentos que, frequentemente, interrompem o processo produtivo e exigem manutenção. A Associação Brasileira de Manutenção, através de um estudo publicado em [1], afirma que, a maior parte dos

\footnotetext{
${ }^{1}$ rodrigoborges@unirv.edu.br

2 fabio.chavarette@unesp.br

3 roberto.outa@fatec.sp.gov.br

${ }^{4}$ michael@unirv.edu.br

${ }^{5}$ darlan@unirv.edu.br
} 
problemas causados em motores de indução são oriundos de falhas em mancais e estatores, que juntas, representam $78 \%$ dos registros efetuados. As falhas relativas a rotores, $10 \%$ dos registros, e $12 \%$ das falhas, se manifestam de forma diversificada.

Segundo [2], a análise e monitoramento de vibrações em máquinas rotativas é muito popular e se apresenta de forma eficaz. O autor ressalta que, a maioria das falhas de equipamentos mecânicos está relacionada a uma mudança na dinâmica das estruturas, e isso ocorre devido a oscilações estruturais do comportamento original. Essas oscilações podem ser utilizadas para identificar presença de comportamentos harmônicos indesejados.

Em [11], destaca-se a importância de identificar precocemente os danos em motores de indução. O autor afirma que, nem sempre, os danos em rotores interrompem o funcionamento dos motores, e isso pode ocasionar desgastes progressivos aos outros componentes, provocando vibrações indevidas, consumo excessivo de corrente e falhas prematuras.

Os sistemas de monitoramento da integridade estrutural (SHM - Structural Health Monitoring) podem ser utilizados para identificar danos em seu estágio inicial, mensurar a gravidade do problema apresentado e intervir na propagação desses problemas, [4]. Segundo [7], os SHMs fazem uso de tecnologias avançadas, como: sensores e técnicas inteligentes, lógica fuzzy e sistemas imunológicos artificiais, a fim de extrair conhecimentos e informações dos processos produtivos, automatizar a tomada de decisões e melhorar a análise dos sinais adquiridos.

Os SHMs, podem ser categorizados através um critério de classificação de danos, de acordo com sua capacidade de abrangência do problema. Inicialmente, utilizavam-se quatro níveis, [9]:

- Nível 1 - Fornece uma indicação quantitativa dos danos;

- Nível 2 - Fornece a localização dos danos;

- Nível 3 - Fornece a quantificação dos danos;

- Nível 4 - Fornece uma previsão da vida útil restante do equipamento.

Posteriormente, [5] acrescentou mais três níveis para essa classificação, todos voltados para a utilização de materiais inteligentes:

- Nível 5 - Estruturas inteligentes para autodiagnóstico do dano estrutural;

- Nível 6 - Controle para formar um sistema de auto reparo estrutural;

- Nível 7 - Controle ativo e estruturas inteligentes para obtenção de um sistema simultâneo de controle e monitoramento.

O algoritmo de seleção negativa, é um modelo computacional inspirado no processo de reconhecimento de padrões, executado pelos sistemas imunológicos biológicos. Essa técnica foi desenvolvida por [3], e baseada no princípio de seleção negativa das células T, que diferenciam as células sadias das infectadas ou danificadas, conhecida como próprias e não-próprias.

As etapas de implementação do ASN compreendem duas fases, [10]:

- Censoriamento:

O fluxograma da figura 1 ilustra as etapas do censoriamento executadas pelo ASN, conforme descrição feita a seguir:

- Inicialmente, define-se o conjunto de cadeias próprias (S), que representam o funcionamento normal do problema (sem defeito); 


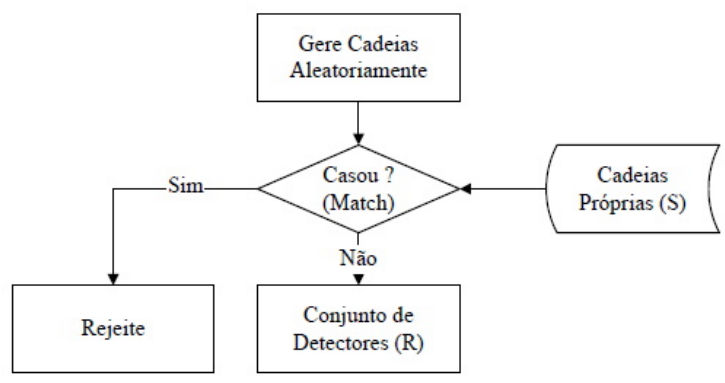

Figura 1: Fluxograma do censoriamento do ASN. fonte: $[8]$.

- São geradas cadeias variadas (aleatoriamente) e verifica-se a afinidade (match) entre elas e as cadeias próprias. Em caso de afinidade superior ao estipulado, rejeita-se a cadeia, caso contrário, essas cadeias passam a compôr o conjunto de detectores, e farão a detecção e classificação de falhas da etapa de monitoramento;

- Monitoramento:

- Nessa fase, monitora-se os dados com intuito de identificar mudanças de comportamento, e então classifica-las de acordo com os detectores. Para isso, compara-se a afinidade de cada uma dessas cadeias protegidas $(S)$ e o conjunto de detectores $(R)$. Sendo a afinidade superior ao limiar estabelecido, um elemento não próprio é identificado e pode ser classificado.

A figura 2 ilustra a etapa de monitoramento executada pelo ASN.

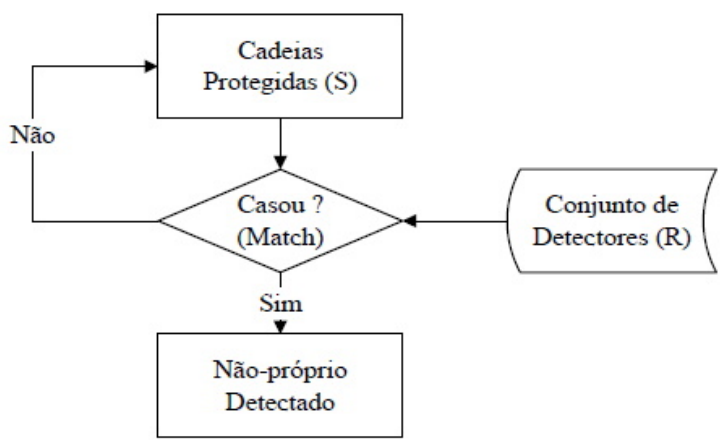

Figura 2: Fluxograma do monitoramento do ASN.

fonte: [8].

Essa pesquisa tem por objeto desenvolver um SHM baseado em técnicas de computação inteligente, a fim de detectar falhas estruturais em sistemas dinâmicos utilizando o algoritmo de sistemas imunológicos artificiais com seleção negativa. 


\section{Metodologia Experimental}

O rotor dinâmico utilizado para aplicação do reconhecimento de padrões e do sistema imunológico desse trabalho, representado na figura 3 , foi baseado no projeto desenvolvido por [6].

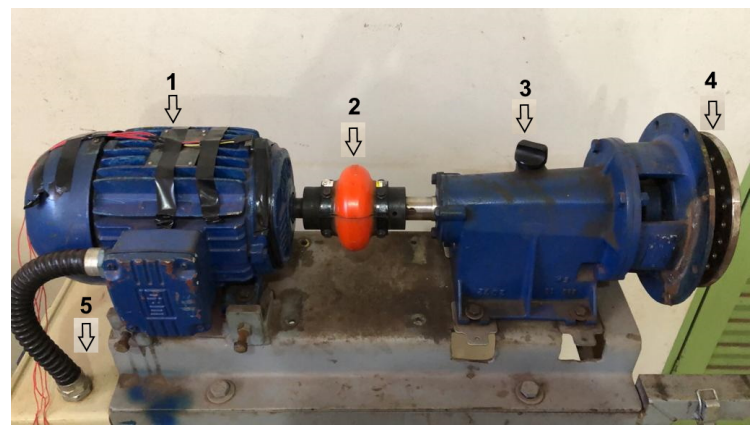

Figura 3: Bancada experimental para aquisição de dados.

A composição da bancada experimental (figura 3), está enumerada e corresponde aos itens:

1. Motor elétrico de $2 \mathrm{HP}$, frequência $60 \mathrm{~Hz}$;

2. Acoplamento flexível Rex Omega tipo Standard;

3. Redutor de velocidade;

4. Disco de aço com 48 furos;

5. Inversor de frequência Schneider Altivar 312 (alternância 0 a $60 \mathrm{~Hz}$ );

Para monitoramento e aquisição dos dados, utilizou-se um microcontrolador Arduíno (modelo Uno) e um acelerômetros MPU6050 de 3 eixos, indicados na figura 4 (a) e (b), respectivamente.
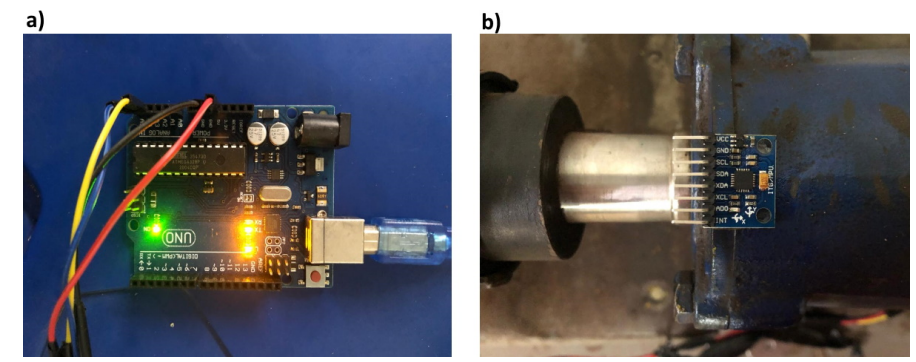

Figura 4: Sistema de aquisição de dados.

Na etapa de monitoramento e aquisição de dados, afim de possibilitar a detecção e caracterização de falhas, procedeu-se as seguintes fases:

- Caracterização dinâmica do sistema (sem adição de massas de desbalanceamento);

- Desbalanceamento provocado pela retirada de parafusos na junta flexível.

Cada fase ocorreu de forma distinta, utilizando-se de algoritmos desenvolvidos com base nos conceitos de: métodos numéricos, sistemas imunológicos e reconhecimento de padrões. 


\subsection{Metodologia de aquisição de dados}

Os dados de vibrações foram coletados através do posicionamento dos acelerômetro sobre o redutor, no ponto A (figura 5), na direção de cada eixo (x, y e z). Cada coordenada cartesiana, gerou um sinal de vibração, com 4000 amostras (aceleração), no tempo de 900 segundos.

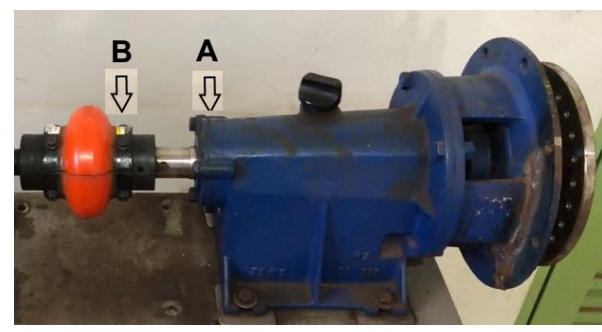

Figura 5: Posicionamento dos sensores.

Para aquisição do banco de dados com sinais livres de falhas (sinais próprios), utilizou-se um intervalo de frequência que variou de $2 \mathrm{~Hz}$ a $60 \mathrm{~Hz}$, em passos de $2 \mathrm{~Hz}$ e 10 sinais para cada frequência. Sendo que, cada sinal de $2 \mathrm{~Hz}$ é composto por 4000 amostras de aceleração.

O banco de dados detectores (não próprios), foi constituído após a retirada de dois parafusos (um oposto ao outro) da junta flexível, no ponto B indicado na figura 5, o que gerou determinado desbalanceamento do sistema. A variação de frequência ocorreu em um intervalo de $2 \mathrm{~Hz}$ a $60 \mathrm{~Hz}$, com passos de $2 \mathrm{~Hz}$ e 10 sinais para cada frequência.

\subsection{Implementação do SHM}

O sistema de diagnóstico de falhas em rotores proposto nesse trabalho, baseou-se no processo de reconhecimento de padrões e aprendizado, realizado no sistema imunológico biológico. Para detecção de falhas, utilizou-se o algorítimo de seleção negativa (ASN).

De posse dos detectores próprios (sinais sem falhas), executou-se o módulo de censoriamento, conforme fluxograma na figura 1, uma vez para cada dano, afim de obter-se o banco de dados do funcionamento com falha. Nessa fase, ocorre o janelamento, ou seja, os dados não-próprios são comparados, janela por janela, com os detectores próprios. Se ocorre o casamento dos dados, o vetor é descartado, caso contrário, detecta-se uma falha e o vetor é armazenado, formando o conjunto de detectores não-próprios. Aqui, são definidos os detectores próprios e não-próprios, que formam o conjunto de detectores e são utilizados para tomada de decisão na fase online.

\subsection{Módulo de monitoramento}

Nessa etapa do SHM é feita a detecção, classificação e diagnostico da falha existente no sistema. No monitoramento online, criou-se um conjunto de dados, que são compostos por todos os sinais da base de dados. Em seguida, selecionou-se aleatoriamente o sinal a ser testado, que foi submetido ao módulo de detecção de novidades. Compara-se esse sinal, com os detectores do ASN, havendo casamento, significa que o sinal é conhecido, sendo direcionado para o modulo de monitoramento. Caso o sinal seja desconhecido (novidade), o SHM não será capaz de realizar o reconhecimento de tal falha, ou seja, quanto maior o banco de dados adquirido pelo processo de censoriamento, mais confiável será o algoritmo. 


\section{Resultados}

O algoritmo correspondente ao SHM, utilizou-se de uma cadeia com 46 sinais normais, capturados de forma randômica do banco de dados de sinais próprios. Foram utilizados 60 do banco de dados com falha, também de modo randômico.

Afim de facilitar a identificação dos picos de ressonância do sistema, é feita a conversão dos dados para o domínio da frequência, através da Transformada Rápida de Fourier. Aplica-se então, o algoritmo de seleção negativa, afim de analisar ponto a ponto a amplitude desses picos de frequência. Os resultados estão representados na tabela 1, considerando-se um desvio padrão de $3 \%$.

Tabela 1: Teste de detecção de falhas.

\begin{tabular}{ccccc}
\hline Total de Sinais & Sinais Próprios & Sinais não Próprios & Taxa de Afinidade & Percentual de Acerto \\
\hline 106 & 46 & 60 & $70 \%$ & $100 \%$ \\
\hline
\end{tabular}

Percebe-se, através dos dados apresentados na tabela 1, que o SHM utilizado apresentou resultados com $100 \%$ de acerto na detecção de falhas.

Afim de expressar a diferença dos sinais próprios e não próprios de maneira gráfica, julgou-se necessário a plotagem desses valores no domínio da frequência, através da aplicação da Transformada Rápida de Fourier (FFT). A frequência utilizada foi $20 \mathrm{~Hz}$, escolhida de forma aleatória, e os resultados podem ser observados na figura 6 .
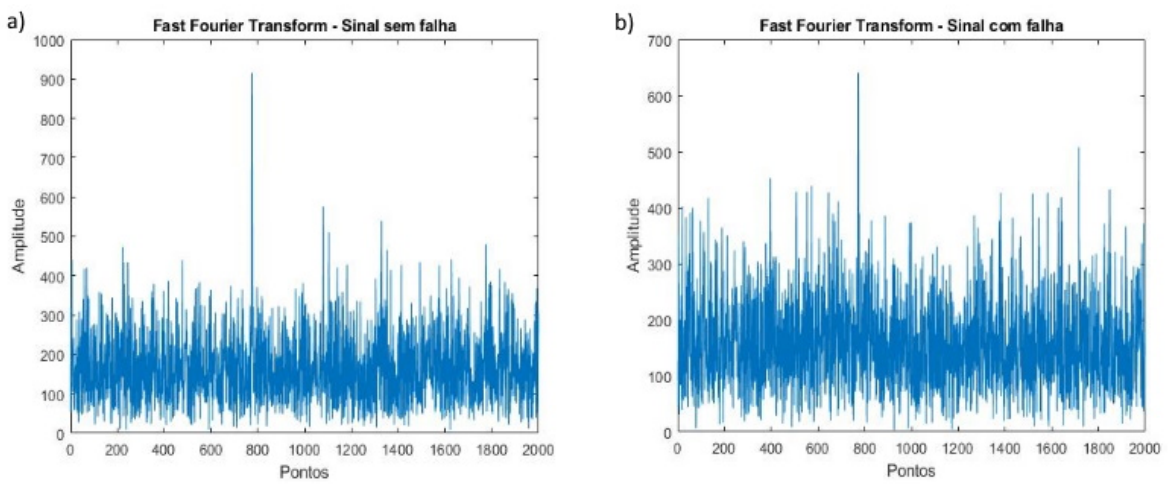

Figura 6: Gráfico no domínio da frequência dos sinais: a) sem falha e; b) com falha.

Os gráficos da figura 6 demonstram notória diferença entre os sinais. No gráfico de sinais com falhas (b), existe maior quantidade de picos de amplitude, quando comparado ao sinal sem falha (a). Com isso, o algoritmo de seleção negativa (ASN) é capaz de identificar e qualificar os sinais não próprios.

\section{Conclusões}

Com base nos critérios de funcionalidade, perante avaliação da precisão e eficiência no processo de diagnóstico de falhas no rotor, o Algoritmo de Seleção Negativa (ASN) apresentou excelente desempenho, quando aplicado para reconhecimento de padrões. Ao comparar-se os sinais próprios e não próprios, o algoritmo foi capaz de identificar as falhas provenientes ao desbalanceamento do sistema, apresentando acertos com $100 \%$ de precisão, conforme demonstrado na tabela 1. 
Diante desses resultados, afirma-se que, a estratégia (detecção, aprendizado e diagnostico) de monitoramento desse algoritmo, pode ser utilizada em máquinas e equipamentos sujeitos a esforços vibracionais, aliando-se as estrategias de manutenção e prevenção de acidentes.

\section{Agradecimentos}

Os autores agradecem ao Laboratório de Sistemas Complexos (Sisplexos) do Instituto de Química - UNESP, por ceder o espaço físico, o parque computacional e ao suporte financeiro da FAPESP, através do processo 2019/10515-4 e ao Conselho Nacional de Desenvolvimento Cientifico e Tecnológico (CNPq), através do processo 312972/2019-9.

\section{Referências}

[1] ABRAMAN, A. situação da Manutenção no Brasil, In: Anais do $26^{\circ}$ Congresso Brasileiro de Manutenção, Curitiba: Abraman, 2011.

[2] Betta, G., Liguori, C., Paolillo, A., Pietrosanto, A. A DSP-based FFT-analyzer for the fault diagnosis of rotating machine based on vibration analysis. IEEE Transactions on Instrumentation and Measurement, v. 51, n. 6, p. 1316-1322, 2002.

[3] Forrest, S., Perelson, A. S., Allen, L., Cherukuri, R. Self-nonself discrimination in a computer, Proceedings of 1994 IEEE computer society symposium on research in security and privacy, p. 202-212, 1994.

[4] Hall, S. R. The effective management and use of structural health data, Proceedings of the 2nd International Workshop on Structural Health Monitoring, p. 265-275, 1999.

[5] Inmam, D. J. Smart structures: example and new problems, Congresso Brasileiro de Engenharia Mecânica - COBREM, n. 16, p. 26-30, 2001.

[6] Jeffcott, H. H. The lateral vibration of loaded shafts in the neighbourhood of a whirling speed - The effect of want of balance, The London, Edinburgh, and Dublin Philosophical Magazine and Journal of Science, volume 37, n.219, p. 304-314, 1919.

[7] Lima, F. P. dos A. Novas Abordagens Híbridas Inteligentes Aplicadas ao Diagnóstico de Falhas Estruturais Utilizando Sistemas Imunológicos Artificiais, Redes Neurais Artificiais e Transformada Wavelet, Tese de Pós Doutorado, UNESP, 2018.

[8] Lima, F. P. dos A. Diagnóstico de distúrbios de tensão em sistemas de distribuição baseado num sistema imunológico artificial com aprendizado continuado, Universidade Estadual Paulista, 2016.

[9] Rytter, A. Vibrational based inspection of civil engineering structures, Dept. of Building Technology and Structural Engineering, Aalborg University, 1993.

[10] Silva, L. N. de C. Engenharia imunológica: desenvolvimento e aplicação de ferramentas computacionais inspiradas em sistemas imunológicos artificiais, Universidade Estadual de Campinas, 2001.

[11] Valles-Novo, R., de Jesus Rangel-Magdaleno, J., Ramirez-Cortes, J. M., Peregrina-Barreto, H., Morales-Caporal, R. Empirical mode decomposition analysis for broken-bar detection on squirrel cage induction motors, IEEE Transactions on Instrumentation and Measurement, v. 64, n. 5, p. 1118-1128, 2014. 